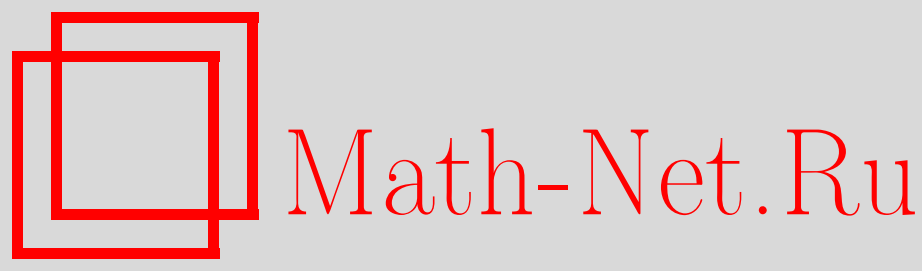

М. И. Монастырский, Поверхности Гекке и преобразования дуальности в решеточных спиновых системах, TMФ, 2010, том 163, номер 3, 505-512

DOI: https://doi.org/10.4213/tmf6518

Использование Общероссийского математического портала Math-Net.Ru подразумевает, что вы прочитали и согласны с пользовательским соглашением http://www . mathnet.ru/rus/agreement

Параметры загрузки:

IP: 54.198 .67 .100

26 апреля 2023 г., $17: 31: 45$

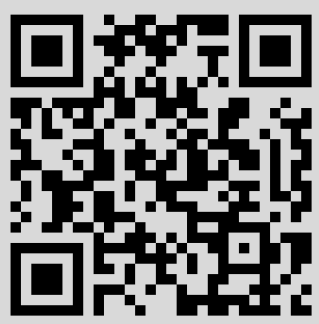




\title{
ФИЗИКА
}

Том 163, № 3

июнь, 2010

2010 г.

М. И. Монастырский*

\section{ПОВЕРХНОСТИ ГЕККЕ И ПРЕОБРАЗОВАНИЯ ДУАЛЬНОСТИ В РЕШЕТОЧНЫХ СПИНОВЫХ СИСТЕМАХ}

\begin{abstract}
Обсуждаются две связанные друг с другом темы: поверхности Гекке и $K$-регулярные графы, с одной стороны, и преобразование дуальности для обобщенных моделей Поттса, с другой стороны. Каждая из них связана с глубокими математическими и физическими теориями, и на первый взгляд они не имеют ничего общего. Однако в последние годы становится все более ясно, что между этими двумя задачами существуют глубокие внутренние связи. Особенно интересна и таинственна в этом контексте роль групп Гекке. Рассмотрены несколько примеров. Изложение имеет в основном описательный характер.
\end{abstract}

Ключевые слова: группа Гекке, модель Поттса, преобразование дуальности.

\section{1. ОСНОВНАЯ КОНСТРУКЦИЯ}

Результаты, представленные в настоящей работе, основаны на статье [1], написанной совместно с моим коллегой Робертом Бруксом, безвременная кончина которого лишила меня прекрасного друга и соавтора. Его идеи о спектральных характеристиках лапласианов на “типичных" римановых поверхностях пока не полностью оценены, но со временем, без сомнения, получат признание.

Пусть Г - конечный $k$-регулярный граф.

ОПРЕДЕЛЕНИЕ 1. Ориентация $\mathcal{O}$ на $Г$ представляет собой приписывание каждой вершине $v \in \Gamma$ циклического порядка ребер, выходящих из $v$. Граф $(\Gamma, \mathcal{O})$ с ориентацией часто называется в литературе жирным графом.

Обобщая конструкцию работы [2], полученную для $k=3$, свяжем с ориентированным графом $(\Gamma, \mathcal{O})$ пару римановых поверхностей $S^{\mathrm{O}}(\Gamma, \mathcal{O})$ и $S^{\mathrm{C}}(\Gamma, \mathcal{O})$, где $S^{\mathrm{O}}(\Gamma, \mathcal{O})$ - риманова поверхность с конечной площадью, а $S^{\mathrm{C}}(\Gamma, \mathcal{O})$ обозначает ее конформную компактификацию. Как и в работе [2], наша идея состоит в том, что спектральная геометрия некомпактой поверхности $S^{\mathrm{O}}(\Gamma, \mathcal{O})$ контролируется (с точностью до геометрических констант) спектральной геометрией ориентированного графа $(\Gamma, \mathcal{O})$,

* Институт теоретической и экспериментальной физики, Москва, Россия.

E-mail: monastyrsky@itep.ru 
который уже можно изучать комбинаторными методами. Спектральная геометрия замкнутой поверхности $S^{\mathrm{C}}(\Gamma, \mathcal{O})$ оказывается близкой к спектральной геометрии открытой поверхности $S^{\mathrm{O}}(\Gamma, \mathcal{O})$, если $S^{\mathrm{O}}(\Gamma, \mathcal{O})$ удовлетворяет условию больших каспов, которое объясняется ниже. Центральным элементом конструкции является следующее определение.

ОПРЕДЕЛЕНИЕ 2. Для заданного $k$ группа Гекке $\mathbf{H}_{k}$ является дискретной подгруппой в $P S L(2, \mathbb{R})$, порожденной матрицами

$$
A_{k}=\left(\begin{array}{cc}
1 & 2 \cos (\pi / k) \\
0 & 1
\end{array}\right), \quad B_{k}=\left(\begin{array}{cc}
0 & -1 \\
1 & 0
\end{array}\right)
$$

Фундаментальная область $F_{k}$ для $H_{k}$ показана на рисунке, где $p_{0}-$ находящаяся в верхней полуплоскости точка пересечения окружностей радиуса 1 с центрами в точках 0 и $2 \cos (\pi / k)$. Замечая, что $i$ - неподвижная точка для $B_{k}$, видим, что $p_{0}-$ неподвижная точка для

$$
A_{k} B_{k}=\left(\begin{array}{cc}
2 \cos (\pi / k) & -1 \\
1 & 0
\end{array}\right)
$$

следовательно, $p_{0}=\cos (\pi / k)+i \sin (\pi / k)$, при этом указанные окружности пересекаются в точке $p_{0}$ под углом $2 \pi / k$.

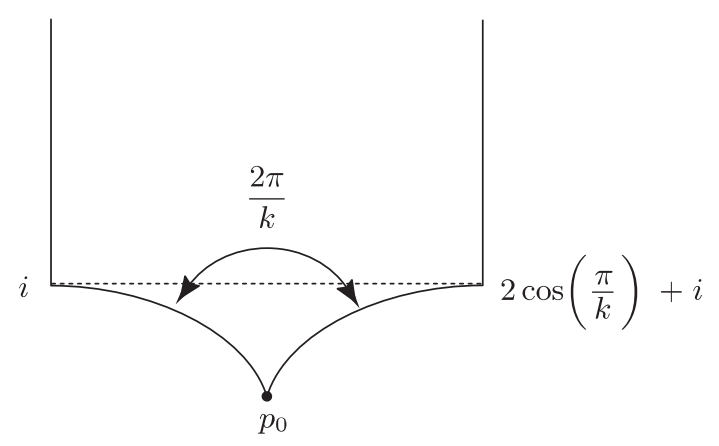

Тот факт, что $A_{k}$ и $B_{k}$ порождают дискретную группу, можно получить из теоремы Пуанкаре о многоугольниках, из того, что $A_{k}$ сохраняет орицикл $y=1$, а также из того, что $A_{k} B_{k}$ представляет собой вращение на угол $2 \pi / k$ вокруг $p_{0}$ и перемещает точку $i$ в точку $2 \cos (\pi / k)+i$. В частном случае $k=3$ имеем $2 \cos (\pi / k)=1$, в результате возникают хорошо известные генераторы и фундаментальная область для $\operatorname{PSL}(2, \mathbb{Z})$.

\section{2. ПОВЕРХНОСТИ ГЕККЕ}

Для каждого $k$ зададим набор $\mathcal{H}_{k}$ поверхностей $S$ таких, что $S=S^{\mathrm{C}}(\Gamma, \mathcal{O})$ для некоторого $k$-регулярного $(\Gamma, \mathcal{O})$. Следующая теорема утверждает, что $\mathcal{H}_{k}$ есть в точности множество $\mathcal{B}$ поверхностей Белого, для которых известно несколько характеризаций [3], [4]. 
Теорема 1 [1]. Для каждого $k$ имеет место равенство $\mathcal{H}_{k}=\mathcal{B}$.

Из этой теоремы, например, следует, что для любой римановой поверхности $S$ и для любого $\varepsilon$ найдется такой $k$-регулярный ориентированный граф $(\Gamma, \mathcal{O})$, что $S^{\mathrm{C}}(\Gamma, \mathcal{O})$ будет $\varepsilon$-близка к $S$ (для любой разумной метрики на пространстве модулей поверхностей). Основной момент здесь состоит в том, что описание поверхности $S$ в виде $S=S^{\mathrm{C}}\left(\Gamma_{k}, \mathcal{O}_{k}\right)$ для некоторого $k$ может быть очень сложным, тогда как для другого $k^{\prime}$ граф $\left(\Gamma_{k^{\prime}}, \mathcal{O}_{k^{\prime}}\right)$ может оказаться достаточно простым.

Отметим, что результат теоремы не является новым, схожая реализация поверхностей Белого приводится в работах [5], [6]. Мы лишь дали новое простое доказательство, связанное с теорией графов.

\section{3. РИМАНОВЫ ПОВЕРХНОСТИ $S^{\mathrm{O}}(\Gamma)$ И $S^{\mathrm{C}}(\Gamma)$}

В данном разделе мы опишем, как извлечь некоторые геометрические свойства поверхностей $S^{\mathrm{O}}(\Gamma)$ и $S^{\mathrm{C}}(\Gamma)$ из комбинаторики графа $\Gamma(G, \mathcal{O})$.

ОПРЕДЕЛЕНИЕ 3. Путь, состоящий из левых поворотов (ПЛП), - это замкнутый путь на $\Gamma(G, \mathcal{O})$ такой, что в каждой вершине путь совершает поворот налево в выбранной ориентации $\mathcal{O}$.

Перемещение по ПЛП на Г представляет собой некий путь на $S^{\mathrm{C}}(\Gamma, \mathcal{O})$, который обходит вокруг каспа. Пусть $l=l(\Gamma(G), \mathcal{O})$ - число ПЛП без общих точек, тогда топология поверхности $S^{\mathrm{O}}(\Gamma, \mathcal{O})$ может быть описана в терминах $l$ и числа вершин $2 n$. Граф $\Gamma$ разбивает $S^{\mathrm{O}}(\Gamma, \mathcal{O})$ на $l$ областей, каждая из которых ограничена неким ПЛП и содержит внутри себя один касп. Используем формулу для эйлеровой характеристики $\chi\left(S^{\mathrm{O}}(\Gamma, \mathcal{O})\right)=2 n-l n+l=2-2 g$. Род поверхности $S^{\mathrm{O}}(\Gamma, \mathcal{O})$ тогда равен $g=1+(n-l) / 2$, а число каспов равно $l$.

ЗАмечАниЕ 1 . Топология поверхности $S^{\mathrm{O}}(\Gamma, \mathcal{O})$ существенно зависит от выбора ориентации $\mathcal{O}$.

Пример 1 [2]. Обычная ориентация на 3-регулярном графе, являющемся 1-скелетом куба, содержит шесть ПЛП, и в качестве соответствующей поверхности мы имеем сферу с шестью проколами, но можно также выбрать два, четыре или шесть ПЛП, так что поверхность может иметь род $0,1,2$.

ПримеР 2. Пусть $(\Gamma, \mathcal{O})$ - ориентированный $k$-регулярный граф, в котором все ПЛП имеют длину $l$. Тогда можно определить дуальный ориентированный граф $\left(\Gamma^{*}, \mathcal{O}^{*}\right)$ как $l$-регулярный граф, в котором все ПЛП имеют длину $k$ : вершины графа $\Gamma^{*}$ суть ПЛП на $\Gamma$, а ребра $\{e\}$ соответствуют ребрам $e$ графа $\Gamma$, причем $\{e\}$ соединяет два (не обязательно различных) ПЛП, которым принадлежит $е$. Ориентация $\mathcal{O}^{*}$ на $\Gamma^{*}$ задается следующим образом: для заданного ПЛП $\gamma$ и ребра $e$ на $Г$ следующий элемент в циклическом порядке в $\{\gamma\}$ есть $\left\{e^{\prime}\right\}$, где $e^{\prime}$ - ребро, следующее за $e$ вдоль пути $\gamma$. 
Интересным примером также является $k$-регулярный платонов граф [1], [7].

Теорема 2 [1]. Имеет место следующее равенство: $S^{\mathrm{C}}(\Gamma, \mathcal{O})=S^{\mathrm{C}}\left(\Gamma^{*}, \mathcal{O}^{*}\right)$.

Описанная выше дуальность связана с двумя типами компактификации поверхности орициклами и с добавлением точек из абсолюта с помощью геодезических, ведущих в каспы. Дело здесь в том, что граф $(\Gamma, \mathcal{O})$ может оказаться сложным для изучения, но дуальный граф $\left(\Gamma^{*}, \mathcal{O}^{*}\right)$ может быть проанализирован достаточно легко. Например, равномерное ограничение снизу постоянной Чигера и первого собственного значения для дуальных платоновых графов $\pi_{k}^{*}$ эквивалентно теореме Сельберга [7] с точностью до константы, но постоянную Чигера и первое собственное значение $\pi_{k}^{*}$ можно вычислить и элементарно [7].

\section{4. БОЛЬШИЕ КАСПЫ}

Геометрию каспов можно также извлечь из структуры $\Gamma(G, \mathcal{O})$. В работе [4] предложена следующая конструкция.

ОПРЕДЕЛЕНИЕ 4. Пусть $S^{\mathrm{O}}$ - риманова поверхность с конечной площадью. Поверхность $S^{\mathrm{O}}$ имеет каспы длины $\gg L$ (выполнено условие болъиих каспов), если имеется система замкнутых орициклов $c_{i}$, такая что:

1) каждый орицикл имеет длину не меньше $L$;

2) каждый касп содержится во внутренности одного из $c_{i}$;

3) внутренности циклов $c_{i}$ попарно не имеют общих точек.

Важность этого условия следует из теоремы, которая утверждает, что если $S^{\mathrm{O}}$ удовлетворяет условию больших каспов, то спектральные геометрии поверхностей $S^{\mathrm{O}}$ и $S^{\mathrm{C}}$ близки друг к другу. Точное утверждение можно найти в работе [4]. Приведем схему доказательства. Если каждый касп имеет орицикл длины по крайней мере $2 \pi$, то можно заклеить данный касп метрикой отрицательной кривизны, конформно изменяя метрику внутри каспа. Ограничение числом $2 \pi$ возникает как необходимое условие из теоремы Гаусса-Бонне. В работе [8] показано, что оно же и достаточно. Соответствующее число для $k$-регулярных графов будет первым целым $m$, для которого $2 m \cos (\pi / k)>2 \pi$. Таким образом, в пределе при стремлении $k$ к бесконечности $m=4$, а при $k=3$ (модулярная группа) $m=7$.

Заметим, что из условия больших каспов не следует, что все замкнутые пути на графе Г являются короткими, это условие относится только к ПЛП. Таким образом, ориентированный граф $(\Gamma, \mathcal{O})$ может иметь много коротких геодезических, но также и каспы длины не меньше $L$ для некоторых больших $L$.

Разумеется, не всегда удобно изменять метрику внутри замкнутых орициклов. Например, в платоновых графах $\pi_{k}$ все ПЛП имеют длину 3 , а потому там нет больших каспов.

ЗАмЕчАние 2. Асимптотическое поведение проявляет себя уже при малых $L$, при $L \sim 7$. Мы обнаружили, что одни и те же критические значения появляются в двух, по-видимому, не связанных друг с другом проблемах - при вычислении статистической суммы в двумерной квантовой гравитации и при анализе случайных блужданий на локально свободных группах. 


\section{5. ГЕОДЕЗИЧЕСКИЕ НА ГРАФАХ И ПОВЕРХНОСТЯХ}

Геодезические на $S^{\mathrm{O}}(\Gamma, \mathcal{O})$ можно описывать в терминах графа $(\Gamma, \mathcal{O})$. Замкнутый путь $P$ длины $k$ на графе можно задавать, начиная со срединной точки на ребре и далее определяя последовательность $\left(w_{1}, \ldots, w_{n}\right)$, где каждое $w_{i}$ есть или $l$, или $\mathrm{r}$, что означает левый или правый поворот в следующей встреченной вершине. Пусть $M_{p}=W_{1} \ldots W_{k}$, где

$$
W_{j}=\left(\begin{array}{cc}
1 & 1 \\
0 & 1
\end{array}\right), \quad \text { если } \quad w_{j}=1 ; \quad W_{j}=\left(\begin{array}{cc}
1 & 0 \\
1 & 1
\end{array}\right), \quad \text { если } \quad w_{j}=\mathrm{r} .
$$

Замкнутый путь $P$ на Г тогда гомотопен замкнутой геодезической $\gamma(P)$ на $S^{\mathrm{O}}(\Gamma, \mathcal{O})$; длину length $(\gamma(P))$ геодезической можно найти из уравнения $\operatorname{ch}[\operatorname{length}(\gamma(P)) / 2]=$ $\operatorname{tr} M_{p}$. Длина геодезической существенно зависит от $\mathcal{O}$. Например, если путь $P$ содержит только левые повороты, то length $(\gamma(P))=0$. Если путь $P$ длины $r$ состоит из чередующихся левых и правых поворотов, то length $(\gamma(P))=r \ln ((3+\sqrt{5}) / 2)$.

ЗАмЕчАниЕ 3. Имеется определенное сходство между вычислением длин геодезических в терминах графов и построением факторов фон Неймана со специальными значениями индекса. Справедлив следующий замечательный результат.

Tеорема 3 [9]. Для каждого целого $k$ существуют фактор $R_{0} \subset R$ и суббактор $R_{0}$ типа $\mathrm{II}_{1}$ с индексом $\left[R: R_{0}\right]$, равным $4 \cos ^{2}(\pi / n)$.

Например, существует фактор с $\left[R: R_{0}\right]=(3+\sqrt{5}) / 2$. Такое сходство не является случайным. Оно связано с конструкцией факторов гиперфинитного типа, использующей диаграммы Брателли. С другой стороны, оно связано с квантованием пространства модулей проверхностей Римана, базирующемся на разложении поверхностей на $k$-валентные графы. Детали мы обсудим в отдельной публикации.

\section{6. ОБОБЩЕННЫЕ МОДЕЛИ ПОТТСА}

В данном разделе мы рассмотрим некоторые связи групп Гекке с моделями Поттса. Кратко напомним определение классической модели Поттса в планарном случае. Подробности приведены в книге [10].

$\mathbb{Z}_{q}$-модель Поттса. Пусть $L$ - двумерная решетка. С каждой вершиной $i$ свяжем "спин" $\sigma_{i}$, принимающий $q$ значений. Два соседних спина $\sigma_{i}$ и $\sigma_{j}$ взаимодействуют с энергией $-J \delta\left(\sigma_{i}, \sigma_{j}\right)$, где $\delta(\cdot, \cdot)$ - дельта-функция Дирака. Полная энергия имеет вид

$$
\mathcal{E}=-J \sum_{(i, j)} \delta\left(\sigma_{i}, \sigma_{j}\right)
$$

где суммирование ведется по всем ребрам $(i, j)$ решетки $L$. Статистическая сумма определяется как

$$
Z_{n}=\sum_{\sigma} \exp \left\{K \sum_{(i, j)} \delta\left(\sigma_{i}, \sigma_{j}\right)\right\}, \quad K=\frac{J}{k_{\mathrm{B}} T},
$$


где $k_{\mathrm{B}}$ - постоянная Больцмана, $T$ - температура, а суммирование ведется по всем значениям спина $\sigma_{i}$. Формула $(2)$ эквивалентна следующей:

$$
Z_{n}=\sum_{\sigma}^{\prime} \prod_{(i, j)}\left(1+v \delta\left(\sigma_{i}, \sigma_{j}\right)\right)
$$

Модель Поттса можно определить и на любом графе $L$.

В работе [11] показано, что $Z_{n}$ для модели Поттса можно выразить в виде дихроматического многочлена, известного в теории графов (см. книгу [12]). Положим $v=e^{K}-1$. Рассмотрим типичный граф $L$, содержащий $l$ связей и $c$ связных компонент (включая изолированные вершины). Пусть $E$ - число ребер графа $L$. Тогда слагаемое в (1) является суммой двух членов, 1 и $v \delta\left(\sigma_{i}, \sigma_{j}\right)$. Поэтому произведение можно разложить в сумму $2^{E}$ слагаемых. Каждый из этих $2^{E}$ членов можно связать с графом на ребрах $L$. Тогда соответствующий член в разложении содержит множитель $v^{l}$. Суммируя по независимым спинам и по всем компонентам, получаем вклад этих членов, равный $q^{c} v^{l}$, где $c$ есть число компонент графа (изолированный узел (вершина) также считается компонентой). Таким образом, статистическая сумма $Z_{n}$ имеет вид суммы по всем графам $G$, нарисованным на $L$. Это выражение называется дихроматическим многочленом или многочленом Уитни-Татта.

В случае антиферромагнетика $K=-\infty$ и $v=-1$, при этом

$$
Z_{n}=\sum q^{c}(-1)^{l}=P_{n}(q)
$$

где $P_{n}(q)$ - хроматический многочлен: ясно, что $P_{n}(q)$ определяет число способов, которыми можно раскрасить узлы решетки $L$, используя $q$ цветов, так, что цвет никаких двух соседних узлов не совпадает. Тем самым $P_{n}(q)$ есть многочлен по $q$, который совпадает со статистической суммой $Z_{n}$ из (4). Важно упомянуть, что выражение (4) определено для любого комплексного числа $q$, не обязательно целого. Имеется замечательная гипотеза о поведении нулей хроматических многочленов.

ГипотезА Бераха. Рассмотрим хроматический многочлен $P_{n}(q)$ для произвольного большого планарного графа. Тогда вещественные нули многочлена $P_{n}(q)$ концентрируются вблизи предельных точек. Эти предельные точки являются так называемыми числами Бераха $q=[2 \cos (\pi / k)]^{2}, k=2,3, \ldots$.

Эта гипотеза все еще не доказана. Имеется интересный подход к ней с использованием квантовых групп [13]. Хотелось бы в общих чертах наметить другой подход, использующий графы Гекке. В этом случае необходимо рассмотреть граф Кэли, порождаемый группами Гекке. Статистическая сумма модели Поттса, определенной на этом графе, сводится к хроматическим многочленам с желаемыми свойствами. Упомянем в заключение, что знаменитая проблема четырех красок на планарном графе в точности эквивалентна свойству, что $P_{n}(4)$ всегда равен нулю. 


\section{7. ЗАКЛЮЧЕНИЕ}

В работе [14] мы обобщили дуальность Крамерса-Ванье на случай, когда спины принимают значения в любой конечной группе, не обязательно абелевой. В работе [15] было дано обобщение на компактные группы. Идея этих работ состоит в том, чтобы вместо соотношения группы $G$, в которой принимают значения спины, и двойственного объекта $\widehat{G}$ изучать пару алгебр, а именно групповую алгебру $[G]$ и пространство $C(G)$ регулярных функций на $G$.

Данный подход представляется вполне естественным с точки зрения квантовых групп. В этой связи интересно исследовать так называемое соответствие Маккея, которое связывает с любой конечной группой $K$ в $S U(2)$ определенный граф, совпадающий с аффинно расширенными диаграммами Дынкина. Вершины этого графа соответствуют неприводимым представлениям группы $K$. Расширенная вершина отвечает одномерному представлению. Недавно в работе [16] эти результаты были распространены на кокомпактные дискретные подгруппы $\gamma$ в $S U(1,1)$. Интересная задача состоит также в рассмотрении соответствия Маккея для групп Гекке.

Последнее, что хотелось бы упомянуть - это соотношение между группами Гекке и двумерной квантовой теорией поля. Эти группы появились как представления монодромии в некоторых группах окрашенных кос и определяли корреляционные функции в $\mathbb{Z}_{3}$-модели Поттса и парафермионной модели Поттса [5], [8], [17]. Как мы уже отмечали, модель Поттса определена не только для целых $q$, можно построить конформную алгебру для любого вещественного $q$. Однако имеется существенная разница между случаями $q \leqslant 4$ и $q>4$ : при $q \leqslant 4$ конформная алгебра является конечномерной, только если $q=[2 \cos (\pi / k)]^{2}$.

Благодарности. Данная статья основана на докладе на Второй международной конференции "Струнная теория поля и смежные вопросы" (Москва, 2009 г.). Я благодарен организаторам за приглашение. Данная работа была закончена во время моих визитов в Institut des Hautes Études Scientifiques (Bures-sur-Yvette, France), Institute for Advanced Study (Princeton, USA) и Mount Holyoke College (South Hadley, USA). Я признателен всем этим учреждениям за гостеприимство и частичную финансовую поддержку.

\section{Список литературы}

[1] R. Brooks, M. Monastyrsky, "K-regular graphs and Hecke surfaces", Geometry, Spectral Theory, Groups, and Dynamics, Contemp. Math., 387, eds. M. Entov, Y. Pinchover, M. Sageev, AMS, Providence, RI, 2005, 65-74.

[2] R. Brooks, E. Makover, J. Diff. Geom., 68:1 (2004), 121-157.

[3] Г. В. Белый, Изв. АН СССР. Сер. матем., 43:2 (1979), 267-276.

[4] R. Brooks, E. Makover, "Belyi surfaces", Entire Functions in Modern Analysis (Tel-Aviv, 1997), Israel Math. Conf. Proc., 15, eds. Y. Lyubich, V. Milman, I. Ostrovskii, M. Sodin, V. Tkachenko, L. Zalcman, Bar-Ilan Univ., Ramat Gan, 2001, 37-46.

[5] P. B. Cohen, C. Itsykson, J. Wolfart, Comm. Math. Phys., 163:3 (1994), 605-627.

[6] G. Jones, D. Singermann, Bull. London Math. Soc., 28:6 (1996), 561-590.

[7] R. Brooks, Comment. Math. Helv., 74:1 (1999), 156-170.

[8] D. Mangoubi, J. Anal. Math., 91:1 (2003), 193-209. 
[9] V.F. R. Jones, Invent. Math., 72:1 (1983), 1-25.

[10] Р. Бэкстер, Точно решаемые модели в статистической механике, Мир, М., 1985.

[11] P. W. Kasteleyn, C. M. Fortuin, J. Phys. Soc. Japan, 26, Suppl. (1969), 11-14.

[12] У. Татт, Теория графов, Мир, М., 1988.

[13] A.B. Zamolodchikov, Exact solutions in two dimensional conformal theory and critical phenomena, Prepint, ITP-87-65, Kiev, 1987.

[14] V. M. Buchstaber, M. I. Monastyrsky, J. Phys. A, 36:28 (2003), 7679-7692.

[15] M. Monastyrsky, Kramers-Wannier Duality for spin systems with non-abelian symmetries, Proc. IV School in Mathematical Physics (Belgrade, 2006).

[16] I. Dolgachev, "McKay's correspondence for cocompact discrete subgroups of SU $(1,1)$ ", Groups and Symmetries, CRM Proc. Lecture Notes, 47, eds. J. Harnard, P. Winternitz, AMS, Providence, RI, 2009, 111-133.

[17] Vl. S. Dotsenko, J. Stat. Phys., 34:5-6 (1984), 781-791. 